\title{
STABLE STATES FOR SUPERCONDUCTING SYSTEMS WITH COMBINED $S$-WAVE AND $D$-WAVE PAIRING. ZERO TEMPERATURE CASE
}

\author{
W. Kumala and R. Gonczarek
}

Institute of Physics, Wrocław University of Technology Wybrzeże Wyspiańskiego 27, 50-370 Wrocław, Poland

(Received September 19, 1997; in final form December 23, 1997)

\begin{abstract}
The Fermi-liquid system with $S$-wave and $D$-wave pairing interaction harmonics is considered. The general equations for energy gap and thermodynamic potential difference for arbitrary temperatures are given. The detailed calculations are performed for zero-temperature limit. The obtained results are illustrated and discussed. The value and structure of energy gap are presented. It is showed that the anisotropic gap can be realized only for $g_{0}<g_{2}$.
\end{abstract}

PACS numbers: $74.20 .-\mathrm{Z}$

\section{Introduction}

Recently, in available experimental information the consensus appears that the Fermi-liquid theory of superconductivity including the modified pairing interaction can be applicable to a broad range of systems of the new generation superconductors, i.e. cuprate, heavy fermions and organic superconductors which reveal existence of the unconventional Cooper pairs (c.f. [1-5]). Though there exist lots of theoretical works, where Fermi systems with modified pairing were considered, most of them involve solely one fixed harmonic in the pairing interaction channel [6-8]. The purpose of this paper is to find the stable states for Fermi-liquid system in which the pairing interaction is represented by the $S$ and $D$ Legendre harmonics precisely. In the present considerations we apply the self-consistent Green function formalism in the frame of the weak-coupling approximation, where the specified pairing harmonics can be easily included [9]. 


\section{Formalism and results}

The BCS gap equation in the case of quite arbitrary pairing interaction can be written as follows:

$$
\Delta_{k}=-(1 / 2) \sum_{k^{\prime}} V_{k k^{\prime}} \Delta_{k^{\prime}} \mathcal{E}_{k^{\prime}}^{-1} \tanh \left(\beta \mathcal{E}_{k^{\prime}} / 2\right)
$$

where

$$
\mathcal{E}_{k}^{2}=\varepsilon_{k}^{2}+\Delta_{k}^{+} \Delta_{k}, \quad \beta=1 /(k T),
$$

and

$$
V_{k k^{\prime}}=\sum_{L} V_{L} P_{L}\left(\widehat{k} \cdot \widehat{k}^{\prime}\right) \theta\left(\hbar \omega-\left|\varepsilon_{k}\right|\right) \theta\left(\hbar \omega-\left|\varepsilon_{k^{\prime}}\right|\right)
$$

where $P_{L}$ are Legendre polynomials and $\omega$ is the characteristic Debye frequency. Assuming that all $V_{L}$ for $L=4,6,8, \ldots$ vanish identically, we investigate the system with a combined $S$-wave and $D$-wave pairing. Replacing the summation over momentum space by integration, Eq. (1) reduces to the following form:

$$
\Delta_{\widehat{k}}=-N(0)\left\langle\left[V_{0}+V_{2} P_{2}\left(\widehat{k} \cdot \widehat{k}^{\prime}\right)\right] \Delta_{\widehat{k}^{\prime}} \Phi\right\rangle,
$$

where

$$
\Phi=\int_{0}^{\hbar \omega} \mathrm{d} \varepsilon_{\widehat{k}^{\prime}} \mathcal{E}_{\widehat{k}^{\prime}}^{-1} \tanh \left(\beta \mathcal{E}_{\widehat{k}^{\prime}} / 2\right)
$$

and

$$
\langle\ldots\rangle=\int \mathrm{d} \Omega_{\widehat{k}^{\prime}} / 4 \pi \cdot \ldots,
$$

$N(0)$ denotes the density of states on the Fermi level per spin and per unit volume. Though the general form of $\Delta_{\widehat{k}^{\prime}}$ should be written as follows:

$$
\Delta_{\widehat{k}}=\sum_{L M} \Delta_{L M} Y_{L M}(\theta, \phi),
$$

where $Y_{L M}$ are spherical functions and $L=0$ or $2,-L \leq M \leq L$, we take into account its reduced form

$$
\Delta_{\widehat{k}^{\prime}}=\Delta\left(S Y_{00}+D Y_{20}\right),
$$

where $\Delta=\left\langle\left|\Delta_{\widehat{k}^{\prime}}\right|\right\rangle, Y_{00}=1, Y_{20}=(\sqrt{5} / 2)\left(3 \cos ^{2} \theta-1\right)$ and

$$
\left\langle\left|S Y_{00}+D Y_{20}\right|^{2}\right\rangle=|S|^{2}+|D|^{2}=1,
$$

which should be the appropriate one as the system favors the most uniform solutions. Although using Eq. (4) we can derive all self-consistent equilibrium states, some of them can be unstable. In order to eliminate them we have to define the thermodynamic potential difference between the paired and normal states. Employing the standard relations [10] and performing an algebra, the thermodynamic potential difference for the case under the discussion can be developed into the equivalent forms

$$
\Delta \Omega=-N(0) \Delta \int_{0}^{S}\left\langle\left|\Delta_{\widehat{k}}\right|^{2} \partial \Phi / \partial \Delta_{\widehat{k}}\right\rangle \mathrm{d} S^{\prime}
$$


or

$$
-\left.5^{3 / 2} N(0) \Delta^{3} \int_{0}^{D}\left|D^{\prime}\right|^{2}\left\langle P_{2}^{3} \partial \Phi / \partial \Delta_{\widehat{k}}\right\rangle\right|_{S=0} \mathrm{~d} D^{\prime}
$$

$$
\begin{aligned}
\Delta \Omega & =-\left.N(0) \Delta^{3} \int_{0}^{S}\left|S^{\prime}\right|^{2}\left\langle\partial \Phi / \partial \Delta_{\widehat{k}}\right\rangle\right|_{D=0} \mathrm{~d} S^{\prime} \\
& -5^{1 / 2} N(0) \Delta \int_{0}^{D}\left\langle\left|\Delta_{\widehat{k}}\right|^{2} P_{2} \partial \Phi / \partial \Delta_{\widehat{k}}\right\rangle \mathrm{d} D^{\prime},
\end{aligned}
$$

where we also include the condition

$$
\left(S D^{*}-S^{*} D\right)\left\langle P_{2} \Phi\right\rangle=0,
$$

which allows us to eliminate the imaginary parts in r.h.s. of Eqs. (10) and (11).

We emphasize, the presented Eqs. (4) and (10) or (11) create possibilities to solve the problem for arbitrary temperatures. However, because of a high degree of complication, in the present paper we restrict our consideration to only the zero temperature case, whereas the results obtained in the opposite limit $T \rightarrow T_{\mathrm{C}}$ can be found in Ref. [11].

Below we also introduce the symbol

$$
g_{L}=\frac{V_{L}}{2 L+1} N(0)
$$

and employ the following formula:

$$
P_{L}\left(\widehat{k} \cdot \widehat{k}^{\prime}\right)=[1 /(2 L+1)] \sum_{M} Y_{L M}^{*}(\widehat{k}) Y_{L M}\left(\widehat{k}^{\prime}\right) .
$$

Inserting $x=\cos \theta$ we can replace the averaging over spherical angles (6) by the integral

$$
\langle\ldots\rangle \rightarrow(1 / 2) \int_{-1}^{1} \mathrm{~d} x \cdot \ldots
$$

In the zero-temperature limit Eq. (5) reduces to the following form:

$$
\Phi=\ln \frac{2 \hbar \omega}{\left|\Delta_{\widehat{k}}\right|} .
$$

Let us note that for $g_{0}>0$ the $\widehat{k}$-isotropic gap $\left(\Delta_{\widehat{k}}=\Delta\right)$ is always the solution of Eq. (4), which reduces to the form

$$
1 / g_{0}=\Phi \text {. }
$$

Equation (4) can be separated to the forms

$$
\left\{\begin{array}{l}
S=g_{0}(1 / 2) \int_{-1}^{1} \mathrm{~d} x\left(S+D Y_{20}\right) \ln \left[2 h \omega /\left(\Delta\left|S+D Y_{20}\right|\right)\right] \\
D=g_{2}(1 / 2) \int_{-1}^{1} \mathrm{~d} x\left(S Y_{20}+D Y_{20}^{2}\right) \ln \left[2 h \omega /\left(\Delta\left|S+D Y_{20}\right|\right)\right]
\end{array} .\right.
$$

Inserting the complex parameters $S$ and $D$ in the forms $S=|S| \exp \left(\mathrm{i} \varphi_{S}\right)$ and $D=|D| \exp \left(i \varphi_{D}\right)$, we get respectively 


$$
\begin{aligned}
& \ln \frac{2 h \omega}{\Delta}-\frac{1}{g_{0}}=\frac{1}{4} \int_{-1}^{1} \mathrm{~d} x\left(1+\left|\frac{D}{S}\right| \exp (-\mathrm{i} \varphi) Y_{20}\right) \\
& \times \ln \left(|S|^{2}+|S D| Y_{20} \cos (\varphi)+|D|^{2} Y_{20}^{2}\right), \\
& \ln \frac{2 h \omega}{\Delta}-\frac{1}{g_{2}}=\frac{1}{4} \int_{-1}^{1} \mathrm{~d} x\left(Y_{20}^{2}+\left|\frac{S}{D}\right| \exp (\mathrm{i} \varphi) Y_{20}\right) \\
& \times \ln \left(|S|^{2}+|S D| Y_{20} \cos (\varphi)+|D|^{2} Y_{20}^{2}\right),
\end{aligned}
$$

where $\varphi=\varphi_{S}-\varphi_{D}$.

Since the r.h.s. of Eqs. (19) must be real, the following conditions have to be fulfilled:

$$
\exp (i \varphi)= \pm 1
$$

or

$$
\begin{aligned}
\int_{-1}^{1} \mathrm{~d} x Y_{20} \ln \left(|S|^{2}+|S D| Y_{20} \cos (\varphi)+|D|^{2} Y_{20}^{2}\right) \\
\quad=\int_{-1}^{1} \mathrm{~d} x Y_{20} \ln \left(|S / D|^{2}+|S / D| Y_{20} \cos (\varphi)+Y_{20}^{2}\right)=0 .
\end{aligned}
$$

In the former case, after integrating we get

$$
\begin{aligned}
& \quad \frac{2 h \omega}{\Delta} \exp \left(-1 / g_{0}\right)=|S+\sqrt{5} D| \\
& \times \exp \left\{-\frac{4}{3}+\frac{\sqrt{5}}{3} \frac{D}{S}+\left(\frac{4}{3}-\frac{2 \sqrt{5}}{3} \frac{D}{S}\right) F\left(\frac{S}{D}\right)\right\} \\
& \frac{2 h \omega}{\Delta} \exp \left(-1 / g_{2}\right)=|S+\sqrt{5} D| \\
& \times \exp \left\{-\frac{16}{15}+\frac{4}{3 \sqrt{5}} \frac{S}{D}+\frac{4}{15}\left(\frac{S}{D}\right)^{2}+\left[\frac{4}{3}-\frac{2}{\sqrt{5}} \frac{S}{D}-\frac{4}{15}\left(\frac{S}{D}\right)^{2}\right] F\left(\frac{S}{D}\right)\right\},
\end{aligned}
$$

where

$$
\begin{aligned}
& F\left(\frac{S}{D}\right)= \\
& \begin{cases}\sqrt{\left(\frac{2}{\sqrt{5}} \frac{S}{D}-1\right) / 3} \arctan \sqrt{3 /\left(\frac{2}{\sqrt{5}} \frac{S}{D}-1\right)} & \text { for } \frac{\sqrt{5}}{2}<S / D \\
\sqrt{\left(1-\frac{2}{\sqrt{5}} \frac{S}{D}\right) / 3} \operatorname{arccoth} \sqrt{3 /\left(1-\frac{2}{\sqrt{5}} \frac{S}{D}\right)} & \text { for }-\sqrt{5}<S / D<\frac{\sqrt{5}}{2} . \\
\sqrt{\left(1-\frac{2}{\sqrt{5}} \frac{S}{D}\right) / 3} \operatorname{arctanh} \sqrt{3 /\left(1-\frac{2}{\sqrt{5}} \frac{S}{D}\right)} & \text { for } S / D<-\sqrt{5}\end{cases}
\end{aligned}
$$


Let us note that dividing (22a) by (22b) we can express the difference $-1 / g_{0}+1 / g_{2}$ as a function of $S / D$

$$
\begin{gathered}
\left(-\frac{1}{g_{0}}+\frac{1}{g_{2}}\right)=\frac{\sqrt{5}}{3} \frac{D}{S}-\frac{4}{15}-\frac{4}{3 \sqrt{5}} \frac{S}{D}-\frac{4}{15}\left(\frac{S}{D}\right)^{2} \\
+\left[-\frac{2 \sqrt{5}}{3} \frac{D}{S}+\frac{2}{\sqrt{5}} \frac{S}{D}+\frac{4}{15}\left(\frac{S}{D}\right)^{2}\right] F\left(\frac{S}{D}\right) .
\end{gathered}
$$

In order to consider the latter case we calculate Eq. (21) numerically. Using the obtained relation between $\cos (\varphi)$ and $|S / D|$ and solving Eqs. (19a) and (19b) we can obtain a relation between $\exp \left(-1 / g_{0}\right) / \Delta$ and $\exp \left(-1 / g_{2}\right) / \Delta$ and $|S / D|$ as a function of $\exp \left(-1 / g_{0}+1 / g_{2}\right)$ (cf. Fig. 3 ).

In order to eliminate unstable states from the obtained results we have to find the thermodynamic potential difference. After including Eq. (16) both Eqs. (10) and (11) reduce to the form

$$
\Delta \Omega=-(1 / 2) N(0) \Delta^{2}\left(|S|^{2}+|D|^{2}\right)=-(1 / 2) N(0) \Delta^{2}
$$

and the condition (12) becomes quite equivalent to Eqs. (20) and (21). We remind that in real physical systems the thermodynamic potential must achieve a minimum, hence merely such states could be realized.

Note that $|S|$ and $|D|$ are dependent. So employing relation (9) we can eliminate real $S$ or $D$ parameter from Eqs. (22)-(24) and r.h.s. of these equations become the functions of only one variable whose variation from -1 to 1 corresponds to a possible pairing state.

In order to derive the value of the energy gap $\Delta$ and its structure (the value of $S$ and $D$ parameters) in dependence on $g_{0}$ and $g_{2}$ we have to prove that Eqs. (19) after including Eqs. (20) and (21) possess a solution.

In Fig. 1 three sets of solutions are presented. The thick-line curve defines values of l.h.s. of Eqs. (22) obtained for the real $D$-parameter varying from -1 to 1 . The straight vertical line marks the isotropic solutions $(S=1, D=0)$ obtained from Eq. (17), which are independent of $g_{2}$. The double-line curve appears as a solution of Eqs. (19) after including Eq. (21) when $S / D$ is the complex number. The distinguished points correspond to the following values $|D|$ and $\cos (\varphi)$, respectively: $\mathrm{A}(1,-1), \mathrm{B}(0.98,-1), \mathrm{C}(0,0), \mathrm{D}(0$, not defined $), \mathrm{E}(0.95,-1), \mathrm{F}(0.79,-1)$, $\mathrm{G}(0, \pm 1), \mathrm{H}(0.19,1), \mathrm{I}(1,1)$.

Moreover we introduce the straight dash line for which we can write the following relation:

$$
\tan \alpha=\exp \left(1 / g_{0}-1 / g_{2}\right)
$$

which is independent of $h \omega$ and $\Delta$. This line crosses the presented plot in a few spots. It means that for the fixed value of the pairing parameters $g_{0}$ and $g_{2}$ several non-equivalent states satisfy the gap equation (1). However, we must remember that the values of $\Delta$ can differ for each state even if $g_{0}$ and $g_{2}$ are fixed. Thus according to Eq. (25) the stable state is the one for which the value of $\Delta$ is the greatest. In Fig. 1 it is marked by $X$. It causes that we can eliminate unstable state solutions from our further consideration and concentrate calculation in the region of the solution given by the $A B C D$ curve. Note that the values of $\triangle$ obtained in 


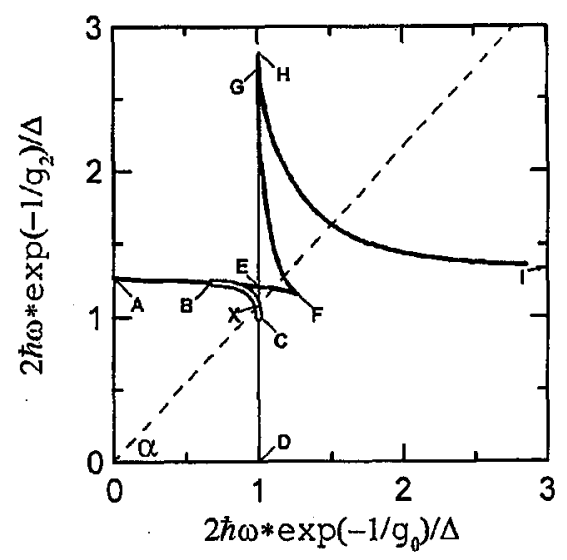

Fig. 1. The regimes of existence of various solutions for the energy gap equation. The solution with $D \equiv 0$ is marked with a thin line, the one with real $S / D$ with a thick line, and the one with complex $S / D$ with a double line.

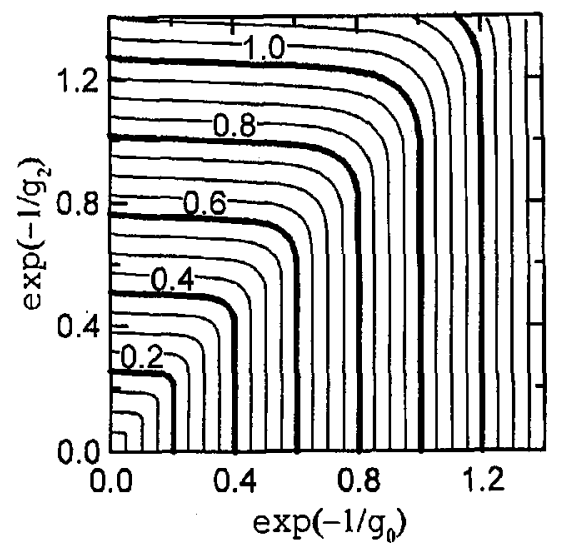

Fig. 2. The equi-energetic lines of the energy gap value $\Delta /(2 h \omega)$ as functions of $\exp \left(-1 / g_{0}\right)$ and $\exp \left(-1 / g_{2}\right)$ in dimensionless units.

the limit cases (BCS and $D$-wave pairing) are in agreement with the well-known results, and we get

$$
\Delta=\gamma_{i}^{-1} 2 h \omega \exp \left(-1 / g_{i}\right)
$$

where $\gamma_{0}=1$ and $\gamma_{2}=1.27$ concern BCS and $D$-wave states, respectively.

In Fig. 2 we present the equi-energetic lines of reduced $\Delta$ for chosen values varying from 0 to above 1 . In order to derive the value of $\Delta$ for the fixed values $g_{0}$ and $g_{2}$ we can employ Eq. (27) where coefficients $\gamma_{i}$ must be estimated, e.g. from Fig. 1. Note that the values of each coefficients $\gamma_{i}$ increase from their limit values to infinity if the other pairing parameter becomes greater.

In Fig. 3 the values of $D$-parameter (thick line) and $\cos (\varphi)$ (thin line) as functions of $\exp \left(-1 / g_{0}+1 / g_{2}\right)$ are presented. Note that the $D$-parameter vanishes 


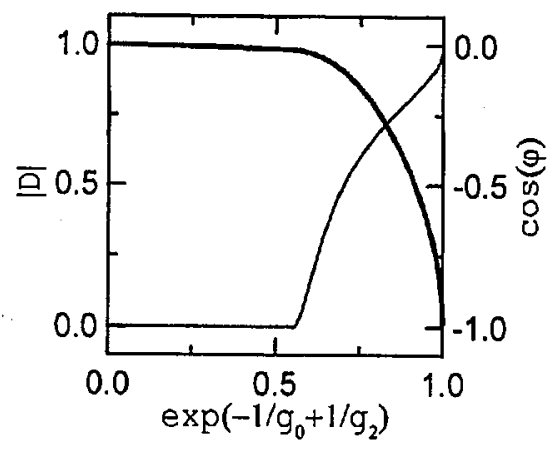

Fig. 3. The value of $|D|$ and $\cos (\varphi)$ as functions of $\exp \left(-1 / g_{0}+1 / g_{2}\right)$. For $g_{2}<g_{0}$ $D \equiv 0$ and $\cos (\varphi)$ is not defined.
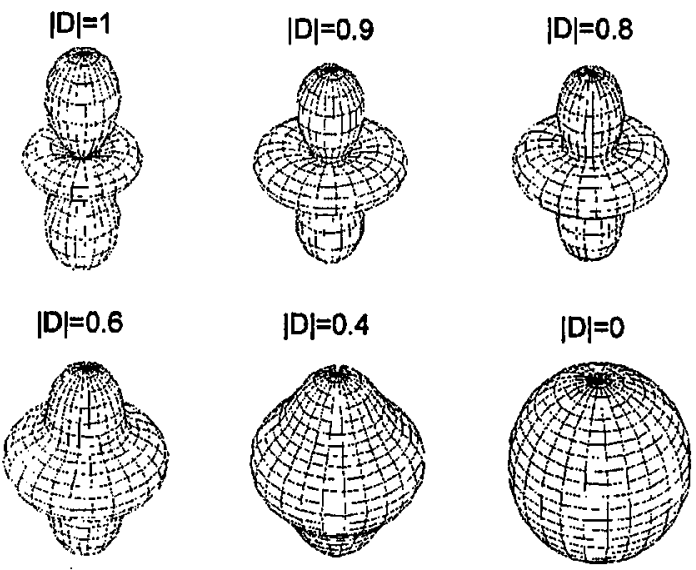

Fig. 4. Exemplary shapes of the energy gap for six decreasing values of $|D|$. Note that the case $|D|=1$ presents the pure $D$-wave state and $|D|=0$ is the isotropic BCS state.

if $g_{2} \leq g_{0}$. So too weak effects of $D$-wave pairing $\left(g_{2} \leq g_{0}\right)$ cannot modify the BCS state.

The above calculations allow us to establish a general form of the energy gap for the case under consideration

$$
\Delta_{k}=\Delta\left[\sqrt{1-D^{2}} \exp (\mathrm{i} \varphi)+|D|(\sqrt{5} / 2)\left(3 k_{z}^{2}-1\right)\right]
$$

where the parameter $\Delta,|D|$ and $\varphi$ can be univocally computed for the fixed $g_{0}$ and $g_{2}$ after employing the results presented in Figs. 2 and 3. In Fig. 4 we exemplify the energy-gap shape $\left(\left|\Delta_{\hat{k}}\right|\right)$ of composed state for six values of $|D|(1,0.9,0.8,0.6$, $0.4,0)$. On the other hand the obtained results permit to derive the pairing parameters $g_{0}$ and $g_{2}$ if we know the value and shape of the energy gap.

Moreover, we stated that some other states could be created in the systems under consideration but they are not stable at $T=0$. That suggests that the 
influence of the temperature causes the enrichment of the energy gap structure. The research in the temperature regime should allow us to find relations between the critical temperature and the other parameters of the system.

\section{Conclusions}

The established relations and presented results reveal influence of the $D$-wave pairing interaction harmonic on a ground state of superconductor. We showed that the isotropic BCS state is ever realized in superconductors if $g_{2} \leq g_{0}$. It means that the $D$-wave pairing interaction harmonic occurs probably in many BCS superconductors but it does not manifest its presence in a ground state. However each modification of a superconducting structure caused by pressure, impurities or geometry can change a relation between $g_{0}$ and $g_{2}$ in such a way that the $S D$-composed state appears.

\section{References}

[1] K.A. Müller, Nature 377, 133 (1995).

[2] N.E. Bickers, D.J. Scalapino, R.T. Scalettar, Int. J. Mod. Phys. B 1, 687 (1987).

[3] R. Heffiner, M. Norman, Comm. Cond. Matt. Phys. 17, 361 (1996).

[4] G. Bruls, D. Weber, B. Wolf, P. Thalmeier, B. Lüthi, A. de Visser, A. Menovsky, Phys. Rev. Lett. 65, 2294 (1990).

[5] S. Adenwalla, S.W. Lin, Q.Z. Ran, Z. Zhao, J.B. Ketterson, J.A. Sauls, L. Taillefer, D.G. Hinks, M. Levy, Bimal K. Garma, Phys. Rev. Lett. 65, 2298 (1990).

[6] D.J. Scalapino, Phys. Rep. 250, 329 (1995).

[7] N. Bulut, D.J. Scalapino, Phys. Rev. Lett. 67, 2898 (1991).

[8] J.R. Schrieffer, Solid State Commun. 92, 129 (1994).

[9] A.J. Leggett, Phys. Rev. A 140, 1869 (1965).

[10] A.L. Fetter, J.D. Walecka, Quantum Theory of Many-Particle Systems, McGraw-Hill, New York 1971, Sec. 51.

[11] P.W. Anderson, W.F. Brinkman, in: The Physics of Liquid and Solid Helium, Eds. K.H. Bennemann, J.B. Ketterson, Part II, John Wiley \& Sons, New York 1978, p. 208. 